\title{
Estudo biomecânico in vitro das suturas em "oito" e de Kessler de tendões flexores de porcos*
}

\section{In Vitro Biomechanical Study on "Figure-of-Eight" and Kessler Sutures in Swine Flexor Tendons}

\author{
Diogo Thomaz Kawachi $^{1}{ }^{10}$ Francisco Simões Deienno ${ }^{1}$ Denis Varanda ${ }^{1}$ Andrea Christina Cortopassi ${ }^{1}$ \\ Trajano Sardenberg ${ }^{1}$ \\ ${ }^{1}$ Departamento de Cirurgia e Ortopedia, Faculdade de Medicina de \\ Botucatu, Universidade Estadual Paulista (Unesp), Botucatu, SP, \\ Brasil \\ Rev Bras Ortop 2020;55(4):445-447. \\ Endereço para correspondência Diogo Thomaz Kawachi, \\ Departamento de Cirurgia e Ortopedia, Faculdade de Medicina de \\ Botucatu, Universidade Estadual Paulista (Unesp), Avenida Professor \\ Mário Rubens Guimarães Montenegro s/n, Campus de Botucatu, \\ Botucatu, SP, 18618687, Brasil (e-mail: thedinhoz@yahoo.com.br).
}

\section{Resumo \\ Palavras-chave \\ - procedimentos ortopédicos \\ - fenômeno biomecânico \\ - traumatismos dos tendões \\ - traumatismos dos dedos \\ - técnicas de sutura}

\section{Abstract}

Objetivo Avaliar as propriedades biomecânicas dos pontos de sutura tendinosa em "oito" e de Kessler.

Métodos Tendões flexores dos dedos de membros superiores de porcos foram divididos em dois grupos com suturas triplas centrais em "oito" (seis passagens) e de Kessler (duas passagens) associadas a suturas periféricas contínuas simples, e submetidos a ensaios mecânicos longitudinais contínuos, obtendo-se as propriedades mecânicas de carga máxima e de energia na carga máxima.

Resultados As médias da carga máxima e da energia na carga máxima na sutura em " 8 " foram de 63,4 N e 217,3 Nmm, respectivamente; na sutura de Kessler, os valores foram de $34,19 \mathrm{~N}$ e $100,9 \mathrm{Nmm}$, respectivamente. A análise estatística indicou que o ponto em "oito" é superior mecanicamente ao ponto de Kessler.

Conclusões Nas condições deste experimento e no tendão flexor de dedo de membro superior de porcos, o triplo ponto em "oito" (seis passagens) é mais resistente do que o ponto de Kessler (duas passagens). O triplo ponto em "oito", com seis passagens, permite movimentação ativa na reabilitação imediata de reparo de tendão flexor de dedo de membro superior com pouco risco de ruptura ou espaçamento na sutura.

Objective To evaluate the biomechanical properties of the "figure-of-eight" and Kessler suture techniques for tendons.

Methods Flexor tendons of porcine fingers were divided into two groups with triple central "figure of eight" sutures (six passages) and Kessler sutures (two passages) associated with simple and continuous peripheral sutures, and submitted to continuous longitudinal mechanical tests, to obtain the mechanical properties of maximum load and energy at maximum load.
* Trabalho Desenvolvido no Departamento de Cirurgia e Ortopedia, Faculdade de Medicina de Botucatu, Universidade Estadual Paulista (Unesp), Botucatu, SP, Brasil. recebido

31 de Março de 2019

aceito

23 de Julho de 2019
DOI https://doi.org/

10.1055/s-0039-1700828. ISSN 0102-3616.
Copyright $\odot 2020$ by Sociedade Brasileira License terms de Ortopedia e Traumatologia. Published by Thieme Revinter Publicações Ltda, Rio de Janeiro, Brazil 


\section{Keywords}

- orthopedic procedures

- biomechanical phenomenon

- tendon injuries

- finger injuries

- suture techniques
Results The mean maximum load and energy at maximum load in the "figure-of- 8 " suture were of $63.4 \mathrm{~N}$ and $217.3 \mathrm{~N}$.mm respectively; in the Kessler suture, the values were of $34.19 \mathrm{~N}$ and $100.9 \mathrm{~N} . \mathrm{mm}$ respectively. The statistical analysis indicated that the "figure-of-eight" suture is mechanically superior to the Kessler technique.

Conclusion Under the conditions of this experiment and in the flexor tendon of porcine fingers, the triple "figure-of-eight" suture (six passages) is more resistant than the Kessler suture (two passages). The "figure-of-eight" suture with six passages enables active movement in the immediate rehabilitation of the flexor tendon repair of the finger, with little risk of rupture or suture spacing.

\section{Introdução}

A necessidade da movimentação ativa nos pós-operatório dos reparos dos tendões flexores dos dedos da mão nas zonas II, III, IV e V, para evitar as aderências e obter amplitude de movimento adequada, exige pontos de sutura com grande resistência mecânica. ${ }^{1,2}$ Entre as várias qualificações do reparo ideal, como número de passadas, qualidades do fio, volume da sutura, entre outras, a facilidade de realização com mínimo trauma cirúrgico é fundamental. ${ }^{2}$ A sutura em "oito" com seis passadas é de fácil realização, pode ser feita com vários tipos de fios cirúrgicos, apresenta grande resistência mecânica para movimentação ativa no pós-operatório, e sua eficiência tem sido comprovada em estudos clínicos e biomecânicos. ${ }^{3-7}$ Apesar de não haver estudos sobre a preferência dos cirurgiões brasileiros em relação ao ponto de sutura dos tendões flexores dos dedos da mão, acredita-se, por observação empírica, que o de Kessler seja um dos mais utilizados. 0 método clássico para estudar as propriedades mecânicas de tendões, íntegros ou suturados, é submeter o corpo de prova a deformações de tensão com velocidade constante. ${ }^{8} 0$ modelo experimental para testar biomecanicamente a sutura imediata de tendões flexores utilizando espécimes de porcos, por teste mecânico de tração longitudinal sob velocidade de tração constante, encontra referência na literatura. ${ }^{9-11} \mathrm{O}$ objetivo do presente estudo foi avaliar biomecanicamente, por meio de teste de tração longitudinal com velocidade constante, a deformação por tensão dos pontos em "oito" e de Kessler em tendões flexores de porcos.

\section{Materiais e Métodos}

Os membros superiores de 18 porcos foram desarticulados no cotovelo, acondicionados em sacos plásticos, e mantidos em freezer na temperatura de -20 graus Celsius. No dia dos experimentos, as peças anatômicas foram descongeladas à temperatura ambiente, e os tendões flexores profundos dos dedos foram dissecados e isolados. Os tendões dos membros superiores direitos foram divididos em dois grupos: grupo 8 (triplo ponto em "8") e grupo K (ponto Kessler). Os tendões dos grupos 8 e $\mathrm{K}$ foram seccionados na região central com lâmina de bisturi número 15 e submetidos a suturas: grupo $8 \mathrm{com}$ triplo ponto em " 8 " com fio monofilamentar de polipropileno 3-0 (Prolene, Ethicon, São José dos Campos, SP, Brasil), e, no grupo K, ponto de Kessler com o mesmo fio cirúrgico; em ambos os grupos houve sutura periférica contínua com fio monofilamentar de polipropileno 4-0 (Prolene) (- Figura 1). Após a sutura, os tendões foram fixados em garras metálicas sinusoidais de alumínio, comprimidas por parafusos com distância de $20 \mathrm{~mm}$ da região da sutura na parte central. As garras foram montadas axialmente em máquina universal de
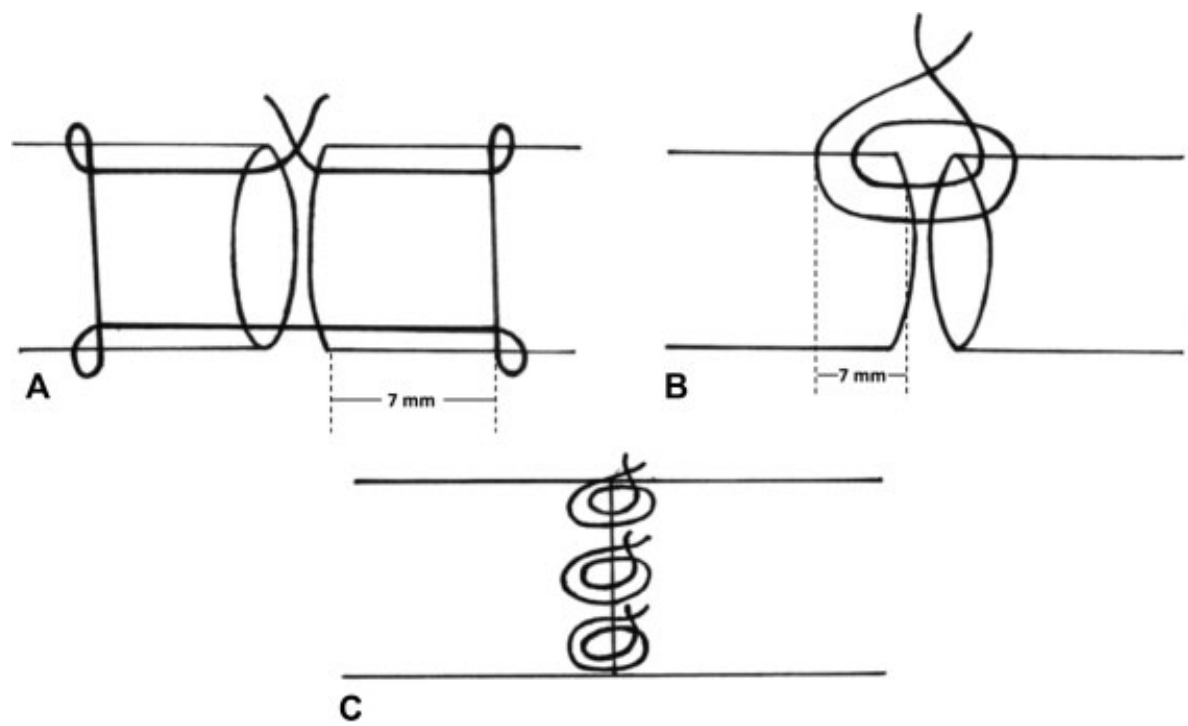

Fig. 1 Representação esquemática das técnicas de reparo de tendão utilizadas nos grupos K (A) e 8 (B e C). 
Tabela 1 Médias, desvios padrões, valores máximos e mínimos e analises estatísticas das propriedades mecânicas de carga máxima $(\mathrm{N})$ e energia na carga máxima ( $\mathrm{Nmm}$ ) dos grupos experimentais Ke 8

\begin{tabular}{|l|l|l|}
\hline Grupos & Carga máxima & Energia na carga máxima \\
\hline $\mathrm{K}(n=8)$ & $\begin{array}{l}34,19 \pm 11,4 ; \\
\text { máximo: } 58,55 ; \\
\text { mínimo: } 18,29\end{array}$ & $\begin{array}{l}100,9 \pm 52,48 ; \\
\text { máximo: } 206,5 ; \\
\text { mínimo } 34,61\end{array}$ \\
\hline $8(n=10)$ & $\begin{array}{l}63,40 \pm 20,40 ; \\
\text { máximo: } 86,04 ; \\
\text { mínimo } 23,17\end{array}$ & $\begin{array}{l}217,3 \pm 93,67 ; \\
\text { máximo: } 365,7 ; \\
\text { mínimo } 33,39\end{array}$ \\
\hline Valor de $p$ & $0,0024^{*}$ & $0,0064^{*}$ \\
\hline
\end{tabular}

Nota: ${ }^{*} \mathrm{p}<0,5$.

ensaio mecânico com célula de carga de $1.000 \mathrm{~N}$ e velocidade de aplicação de $30 \mathrm{~mm} / \mathrm{min}$ (EMIC DL 10000, Instron, São José dos Pinhais, PR, Brasil). Após o ensaio, o computador acoplado à máquina forneceu as propriedades mecânicas de carga máxima $(\mathrm{N})$ e de energia na carga máxima (Nmm).

As análises estatísticas dos resultados foram realizadas com o teste $t$ de Student, com valores de significância para $p<0,5$.

\section{Resultados}

Nos grupos 8 e K, as rupturas ocorreram sempre na área da sutura, não sendo possível determinar a sequência dos pontos rompidos, uma vez que não houve filmagem dos ensaios mecânicos. A - Tabela 1 apresenta os resultados das propriedades mecânicas nos dois grupos, os quais indicam valores maiores para o grupo $8(p<0,5)$.

\section{Discussão}

O presente estudo demonstrou que o ponto em "oito", na configuração de seis passadas, ou seja, feito três vezes, apresenta valores das propriedades mecânicas de carga máxima e energia na carga máxima maiores estatisticamente do que os do ponto de Kessler, resultados semelhantes aos do estudo de de Al-Qattan e Al-Turaiki. ${ }^{3}$ o valor da carga máxima de uma sutura de tendão flexor de dedos de membro superior para permitir movimentação ativa sem risco de ruptura ou formação de espaçamento na sutura é de pelo menos $40 \mathrm{~N}$, valor maior do que o observado no grupo $\mathrm{K}(34,19 \mathrm{~N})$ e inferior ao do grupo 8 $(63,4 \mathrm{~N})$, indicando segurança do triplo ponto em " 8 ". 12

O conhecimento da propriedade mecânica carga máxima é fundamental na avaliação da resistência de determinada sutura de tendão, sendo um dos parâmetros mais utilizados em estudos biomecânicos. ${ }^{2,8,12}$ Por outro lado, a importância clínica da propriedade energia na carga máxima não está totalmente esclarecida. ${ }^{2}$ A energia na carga máxima representa a capacidade de absorção de impacto de determinado material; o valor maior dessa propriedade poderia, em tese, amenizar o impacto da sutura no sistema de polias do canal osteofibroso na região anterior dos dedos durante a movimentação articular, facilitando o deslizamento do tendão e dificultando a formação de aderências cicatriciais.
O presente estudo apresenta limitações metodológicas: a não realização das mensurações da carga necessária para produzir um espaçamento na sutura que, em tese, pode prejudicar a cicatrização; a utilização de ensaio mecânico contínuo e longitudinal, em vez de testes cíclicos e curvilíneos; e, por fim, o uso de tendões isolados de porco, em vez de tendões humanos de mãos ou dedos. Contudo, apesar das limitações inerentes aos métodos utilizados, deve-se ter em mente que a base central do estudo foi a comparação das propriedades mecânicas imediatas das suturas em "oito" e de Kessler, ambas realizadas e testadas nas mesmas condições experimentais e, portanto, os resultados obtidos apresentam validade científica.

\section{Conclusão}

Nas condições deste experimento e no tendão flexor de dedo de membro superior de porco, o triplo ponto em "oito" de AlQattan e Al-Turaik ${ }^{3}$ (seis passagens) é mais resistente do que o ponto de Kessler (duas passagens). O ponto em “oito" com seis passagens permite a movimentação ativa na reabilitação imediata de reparo de tendão flexor de dedo de membro superior com pouco risco de ruptura ou espaçamento na sutura.

Conflito de Interesses

Os autores declaram não haver conflito de interesses.

\section{Referências}

1 Bindra RR. Basic pathology of the hand, and forearm: tendon and ligament. In: Berger RA, Weiss AP, editors. Hand surgery. Philadelphia: Lippincott Williams \& Wilkins; 2003:23-35

2 Pérez-Barquero JÁ, Sabaté EF, Sánchez-Alepuz E. Concepto biomecánicos de las suturas tendinosas. Rev Iberam Cir Mano 2018; 46:143-149

3 Al-Qattan MM, Al-Turaiki TM. Flexor tendon repair in zone 2 using a six-strand 'figure of eight' suture. JHand Surg Eur Vol 2009;34 (03):322-328

4 Al-Qattan MM. Finger zone II flexor tendon repair in children (5-10 years of age) using three 'figure of eight' sutures followed by immediate active mobilization. J Hand Surg Eur Vol 2011;36(04):291-296

5 Al-Qattan MM.Zone 2 lacerations of both flexor tendons of all fingers in the same patient. J Hand Surg Eur Vol 2011;36(03):205-209

6 Agrawal AK, Mat Jais IS, Chew EM, Yam AK, Tay SC. Biomechanical investigation of 'figure of 8' flexor tendon repair techniques. J Hand Surg Eur Vol 2016;41(08):815-821

7 Al-Thunayan TA, Al-Zahrani MT, Hakeem AA, Al-Zahrani FM, AlQattan MM. A biomechanical study of pediatric flexor profundus tendon repair. Comparing the tensile strengths of 3 suture techniques. Saudi Med J 2016;37(09):957-962

8 Nordin M, Lorenz T, Campelo M. Biomecânica de tendões e ligamentos. In: Nordin M, Frankel VH, editors. Biomecânica básica do sistema musculoesquelético. Rio de Janeiro: Guanabara Koogan; 2003:86-107

9 Viinikainen A, Göransson H, Huovinen K, Kellomäki M, Rokkanen P. A comparative analysis of the biomechanical behaviour of five flexor tendon core sutures. J Hand Surg [Br] 2004;29(06):536-543

10 Xie RG, Tang JB. Investigation of locking configurations for tendon repair. J Hand Surg Am 2005;30(03):461-465

11 Cao Y, Zhu B, Xie RG, Tang JB. Influence of core suture purchase length on strength of four-strand tendon repairs. JHand Surg Am 2006;31(01):107-112

12 TangJB, Xie RG. Biomechanics of core and peripheral tendon repairs. In: Tang JB, Amadio PC, Guimberteau JC, Chang J, editors. Tendon surgery of the hand. Philadenphia: Saunders Elsevier; 2012:35-48 